\title{
Giving antioxidants a bad rap
}

Normal cells lacking attachment to the extracellular matrix (ECM) commonly undergo an apoptotic programme termed anoikis, which can be prevented by many oncogenes. However, autophagy can also be induced in unattached cells. As autophagy is a catabolic process often induced under conditions of energy deprivation, and inhibition of apoptosis does not prevent death of ECM-deprived cells, Zachary Schafer, Joan Brugge and colleagues proposed that ECM attachment might regulate cellular metabolism.

Using MCF-10A and primary human mammary epithelial cells, they showed that the loss of ECM attachment reduced ATP levels independently of apoptosis or autophagy. In MCF-10A cells, this ATP reduction was prevented by ERBB2 expression, which stabilized epidermal

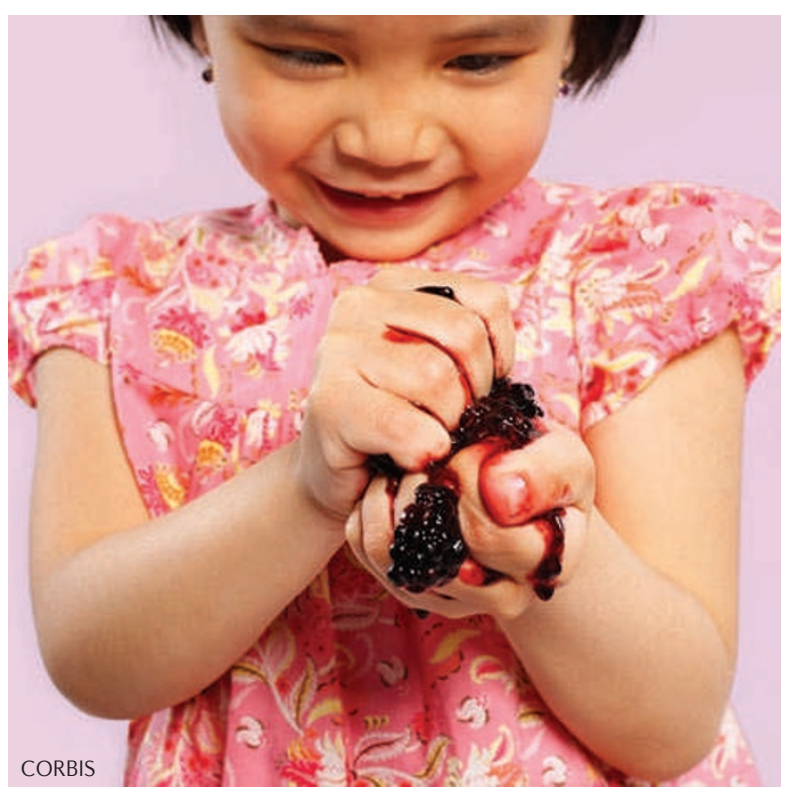

growth factor receptor (EGFR). PI3K (but not Erk) inhibition blocked the ERBB2-induced rescue of ATP levels, and constitutively active variants of either PI3K or AKT1 were sufficient to increase ATP levels in detached cells.

How does PI3K-Akt signalling increase ATP levels? Consistent with the role of this pathway in glucose transport, Schafer et al. found that glucose uptake was reduced in detached cells and restored by ERBB2 expression in a PI3K-dependent manner. Furthermore, administration of a glucose analogue that inhibits glycolysis prevented the ERBB2-induced increase in ATP levels, suggesting that the PI3Kdependent glucose uptake is crucial for ATP production.

Glucose can also be used by the pentose phosphate pathway to produce the reducing agent $\mathrm{NADPH}$ and to decrease levels of reactive oxygen species (ROS), so the authors investigated the role of ECM attachment in ROS production. Detached cells had increased levels of ROS, and this was prevented by ERBB2 expression. Pentose phosphate pathway inhibitors blocked the ERBB2-induced increase in ATP, presumably through the reduction of ROS levels. Therefore, the authors also examined the effects of antioxidants (which also reduce levels of ROS) on detached cells and found that antioxidant treatment increased ATP production through increased fatty acid oxidation.

To examine this pathway in a model with more physiological relevance, the authors used MCF-10A three-dimensional cultures. As cells proliferate in these cultures they form solid spheres, with loss of ECM attachment in inner cells by day 4 , and cell death to clear the lumen starting at day 8 . At day 8, lower levels of NADH and NADPH native fluorescence (a metabolic reporter) were observed in inner cells than in outer cells, and at day 7 ROS were detectable in the inner cells only. Antioxidant treatment abolished the differences in NADH and NADPH fluorescence between the inner and outer cells, and impaired luminal clearance, indicating that reducing ROS levels can promote cell survival. Can antioxidants also promote cell transformation? MCF-10A cells expressing oncogenes that promote proliferation and suppress apoptosis (either human papillomavirus E7 and BCL-2, or ERBB2) exhibit limited colony formation in soft agar, but antioxidant treatment increased both the number and the size of colonies.

This study highlights two mechanisms by which metabolic defects caused by ECM detachment can be rescued: expression of an oncogene that permits glucose uptake in unattached cells, and neutralization of ROS, which allows ATP production through fatty acid oxidation. In addition, antioxidants may promote tumours by suppressing the ability of ROS to prevent outgrowth of cells that are displaced from their natural microenvironment.

Sarah Seton-Rogers

ORIGINAL RESEARCH PAPER Schafer, Z. T. et al. Antioxidant and oncogene rescue of metabolic defects caused by loss of matrix attachment. Nature 19 Aug 2009 (doi:10.1038/nature08268) 